\title{
Symptomatic Superficial Siderosis due to an Intracranial Arteriovenous Malformation
}

\author{
Nancy McLaughlin, Michel W. Bojanowski
}

Can. J. Neurol. Sci. 2007; 34: 386-389

Superficial siderosis (SS) is a rare disorder resulting from subclinical recurrent or continuous bleeding into the subarachnoid space. Only a small portion of patients present the distinct clinical syndrome characterized by sensorineural deafness, cerebellar ataxia, and myelopathy with pyramidal signs. ${ }^{1,2}$ In approximately $60 \%$ of cases the source of hemorrhage is identified. Most commonly, dural pathologies and tumors have been responsible of SS. Vascular abnormalities have been less frequently reported. ${ }^{2}$

Of the 16 cases of intracranial vascular malformations associated with SS, only four have been attributed to a cerebral

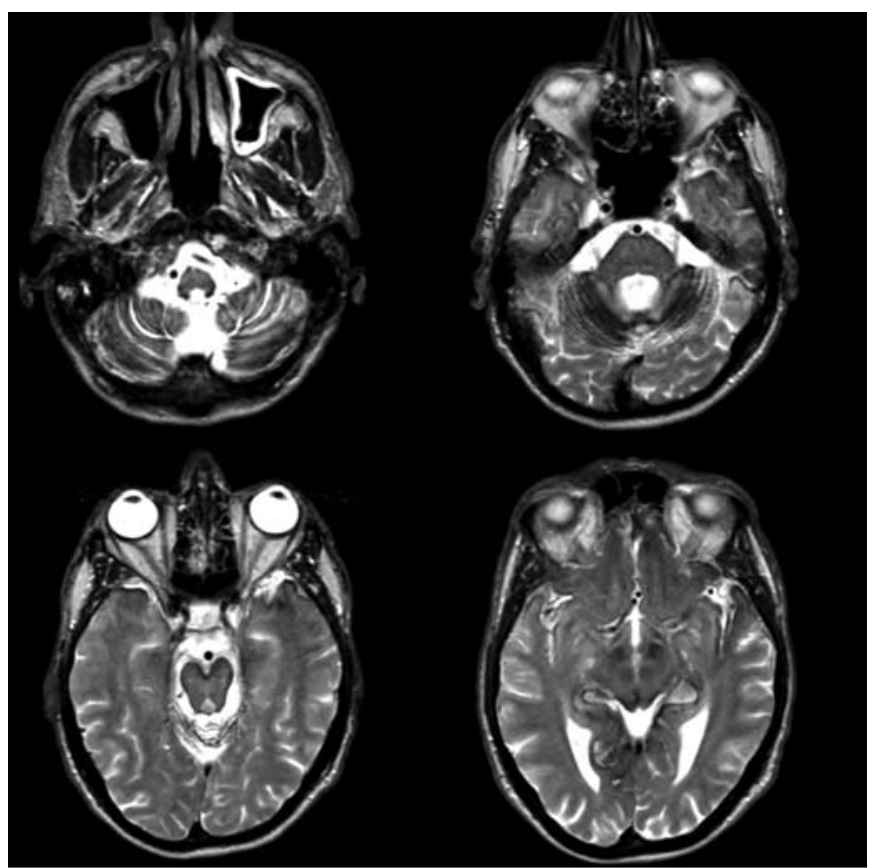

Figure 1. Cranial axial T2-weighted MRI demonstrating a hypointense rim of hemosiderin deposition along the pial surface of cortical gyri, sylvian fissures, cerebellum, brainstem, and cranial nerves. arteriovenous malformations (AVM). Of these, two cases were asymptomatic and two, symptomatic. ${ }^{3-6}$ All were diagnosed using imaging and one patient had surgical resection of the AVM. Until presently, the factors rendering an AVM susceptible to develop SS remain unknown. ${ }^{4}$

We describe a patient who presented typical clinical features of SS. Characteristic hemosiderin deposition was revealed by magnetic resonance imaging (MRI). An orbito-frontal AVM was identified by cerebral angiography and was surgically resected. In the literature, only one other case of symptomatic SS attributable to an AVM has been treated surgically. Reported characteristics of the four cases of AVM associated with SS were reviewed.

A 41-year-old male was referred to our neurosurgical service by colleague neurologists who were following the patient for seizures. In his youth, the patient reported a history of closed head injury not requiring hospitalization. Since the age of 25 , the patient presented a progressive hearing loss. Investigation established a sensorineural deafness without explanation. In the following years, the patient noted progressive gait disturbances. At 37-years-old he presented de novo partial motor seizures and was referred to the neurologist. The initial cerebral computed tomography (CT) showed significant cortical and cerebellar atrophy. No other abnormality was noted. Antiepileptic medication initially controlled the epilepsy. However, two years later the seizures increased in frequency in spite of numerous medication adjustments. In addition, the ataxia became more pronounced. An MRI confirmed significant cortical and cerebellar atrophy. The T2-weighted images revealed a superficial hypointense rim along the pial surface of cortical gyri, sylvian fissures, cerebellum, brainstem, and cranial nerves (Figure 1). Gradient echo and MPGR sequences had not been

From the Division of Neurosurgery, Department of Surgery, Centre Hospitalier de l'Université de Montréal - Hôpital Notre-Dame, Montreal, QC, Canada. Received February 6, 2007. AcCePted in FinAl Form April 29, 2007. Reprint requests to: Michel W. Bojanowski, Neurosurgery Division, CHUM-Hôpital Notre-Dame, 1560 Sherbrooke St. East, Montreal, Quebec, H2L 4M1, Canada. 


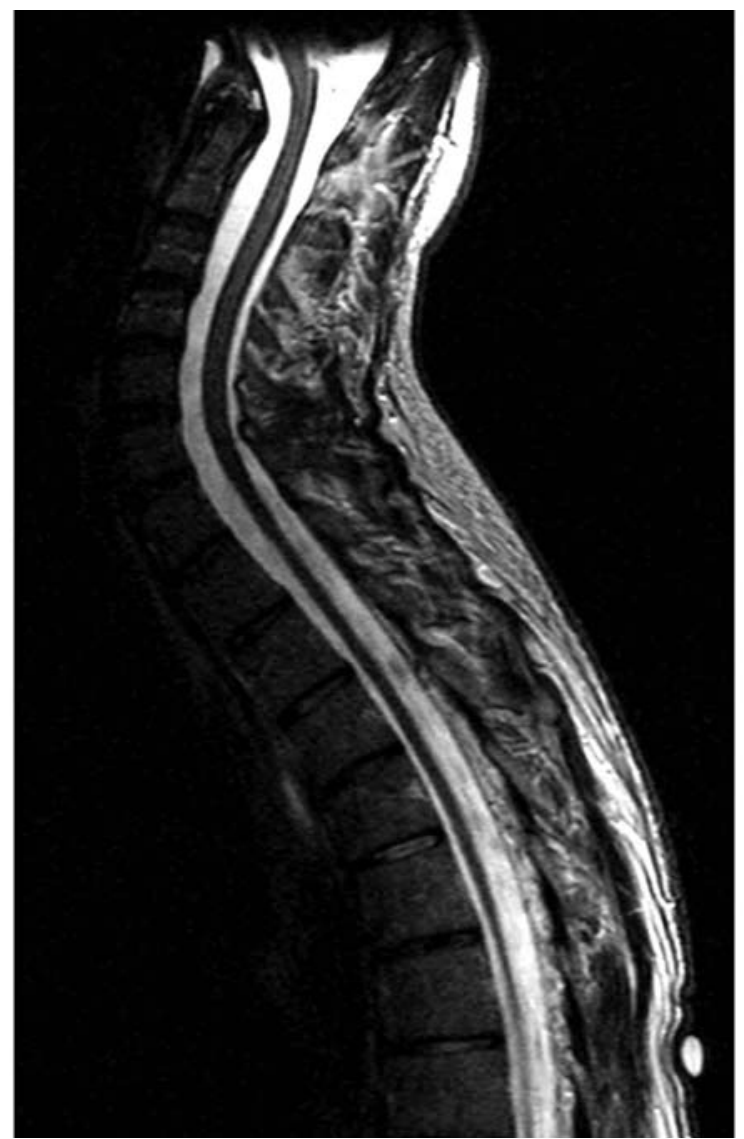

Figure 2. Spinal sagital T2-weighted MRI revealing a hypointense rim surrounding the spinal cord.

performed. Angio-MRI revealed a left orbito-frontal arterial branch that appeared larger than its right counter part. Spinal MRI showed a hypointense rim surrounding the spinal cord (Figure 2). Cerebral angiography demonstrated a left superficial frontal AVM, measuring less than 2 centimeters, supplied by M2 arteries and drained by an orbito-frontal vein joining the superior sagital sinus and the vein of Labbé towards the transverse sinus (Figure 3). These findings suggested that SS was due to recurrent hemorrhage of the AVM. At the time of consultation in neurosurgery, the gait disturbance had progressed and urinary disturbances had appeared. Neurological examination revealed anosmia, dysarthric speach, bilateral sensorineural deafness, prominent gait ataxia, paraparesis, spasticity and hyperreflexia in lower extremities. Surgical resection of the AVM was proposed to remove the source of recurrent bleeding.

A left frontal craniotomy was performed and the diffusely thickened dura was reflected. The entire cerebral cortex visualized through the craniotomy presented a yellowish coloration, more pronounced in the subarachnoid spaces adjacent to and overlying the AVM. This finding supported the hypothesis

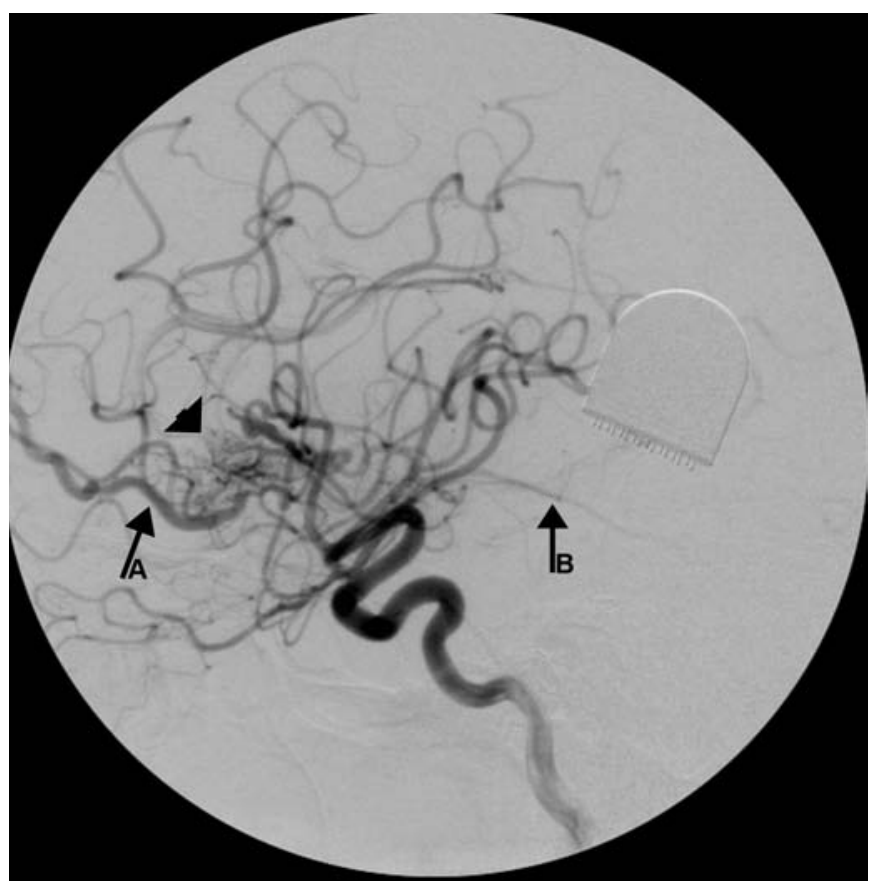

Figure 3. Lateral view of cerebral angiography after left internal carotid artery injection revealing a left superficial frontal AVM (arrow head), measuring less than 2 centimeters and supplied by $M 2$ arteries and drained by an orbito-frontal vein (Arrow A) joining the superior sagital sinus and the vein of Labbé (Arrow B) towards the transverse sinus.

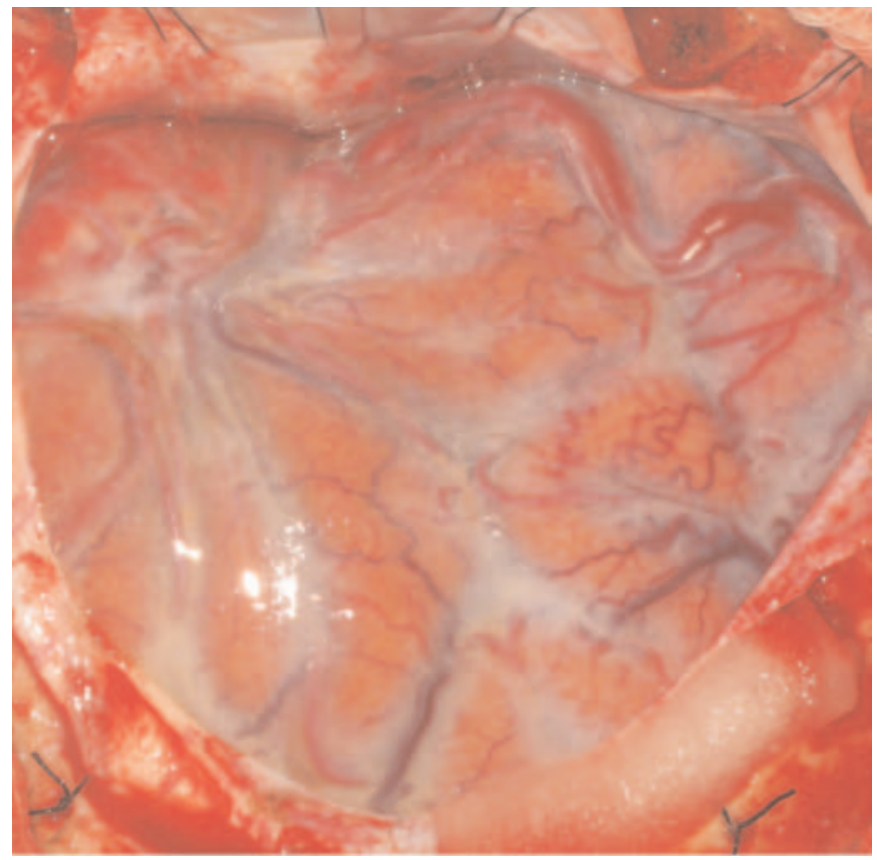

Figure 4. Intra-operative view. The entire cerebral cortex visualized through the craniotomy presented a yellowish coloration, more pronounced in proximity to the AVM. 
that the AVM was the source of hemorrhage (Figure 4). No intraoperative bleeding from the vascular malformation was observed during surgery. The AVM was completely resected as demonstrated by the post-operative angiography. The postoperative course was favorable, the patient reporting gait improvement, diminished spasticity and decreased urinary disturbances in the weeks following surgery.

\section{Discussion}

Superficial siderosis results of repeated asymptomatic episodes of bleeding in the subarachnoid spaces with subsequent deposition of hemosiderin in the subarachnoid and subpial spaces. The deposition of hemosiderin is associated with neuronal loss, gliosis and demyelinisation, ${ }^{2}$ which may largely explain this patient's significant cortical and cerebellar atrophy. The source of hemorrhage is found in approximately $60 \%$ of cases including dural pathologies, tumors and less frequently vascular malformations. ${ }^{2}$

Although asymptomatic in most cases of SS, the subarachnoid hemorrhage may present with sudden onset of severe headache in 14 to $37 \%$ of patients. ${ }^{1,2}$ Patients with SS present a pre-symptomatic phase during which SS is present, but not sufficient to cause symptoms, varying from 4 months to 30 years. $^{2}$ When SS becomes symptomatic, the most frequent clinical features are hearing impairment, cerebellar ataxia and a myelopathic syndrome with pyramidal signs. ${ }^{1,2}$ Less common findings include dementia, bladder disturbances and sensory deficits. Cranial nerves other than the VIIIth may infrequently be involved. The following clinical signs resulting from cranial nerve dysfunction have been previously reported: anosmia, blurring of vision, papillary anisocory, abnormal light reflex, extraocular nerve palsies, trigeminal sensory loss, and facial palsy. $^{2}$ Most frequently, as in our patient, symptoms install progressively over many years, emphasizing the insidious nature of SS. ${ }^{2,7}$

Fearnley and colleagues reported that $75 \%$ of symptomatic patients had an abnormal CT scan. Cerebellar atrophy occurred in nearly $50 \%$ of cases. ${ }^{2}$ A hemosiderin subpial rim, a specific finding for SS, was found in only $20 \%$. $^{2}$ Magnetic resonance imaging has enabled pre-mortem diagnosis of SS. Diffuse hypointensity at the surface of the cerebellum, brainstem, cerebral hemispheres and spinal cord on T2 weighted images is pathognomonic of SS. , $^{2,7}$

Including the present report, 16 cases of intracranial vascular malformations have been associated with SS in the literature (Table). Superficial siderosis was attributed to a venous malformation in one case, ${ }^{8}$ an aneurysm in 3 cases,,${ }^{9,10}$ a cavernous angioma in 7 cases, ${ }^{11-14}$ an AVM in five cases. ${ }^{3-6}$ The AVMs' were located at the ventricular or paraventricular region in two cases ${ }^{3,5,6}$ and at the cerebral convexity in two other cases. ${ }^{4}$ The location was not detailed for one patient. ${ }^{3}$ Including our case, AVMs' size was detailed in two cases, both presenting a diameter less than 2 centimeters. ${ }^{4}$ Until presently, no risk factors have been identified to predict which AVM may be more susceptible to develop SS. Multiple natural history AVM studies

Table: Intracranial Vascular pathologies associated with superficial siderosis

\begin{tabular}{lll}
\hline Vascular malformation & Author (Year) & Location \\
\hline \hline Aneurysm & Cammermeyer (1947) & Vertebral artery \\
Aneurysm & Hughes, Oppenheimer (1969) & Anterior communicating artery \\
Aneurysm & Hughes, Oppenheimer (1969) & Middle cerebral artery \\
Posterior fossa AVM & Koeppen, Dentinger (1988) & Inferior medullary velum \\
Posterior fossa AVM & Parnes, Weaver (1992) & Inferior medullary velum (same case as 5a) \\
Cerebral AVM & Offenbacher et al. (1996) & Lateral ventricle \\
Cerebral AVM & Offenbacher et al. (1996) & Not specified \\
Cerebral AVM & Haroun et al. (2000) & Cerebral convexity, Frontal lobe \\
Cerebral AVM & Present case & Cerebral convexity, Frontal lobe \\
& McLaughlin, Bojanowski (2007) & \\
Cavernous angioma & Hashimoto, Hoyt (1996) & Lateral ventricle \\
Cavernous angioma & Hsu et al. (1999) & Brainstem, cerebral and cerebellar hemispheres \\
Cavernous angioma & Li et al. (2001) & Brainstem, cerebral and cerebellar hemispheres \\
Cavernous angioma & Li et al. (2001) & Brainstem, cerebral and cerebellar hemispheres \\
Cavernous angioma & Li et al. (2001) & Brainstem, right cerebral hemisphere \\
Cavernous angioma & Leussink et al. (2003) & Brainstem, cerebral hemispheres \\
Cavernous angioma & Leussink et al. (2003) & Brainstem, cerebral hemispheres \\
Venous malformation & McGee et al. (1962) & Thalamus - peri-ventricular \\
\hline
\end{tabular}


have found a relationship between the size of AVMs and the frequency of hemorrhage: small AVMs seem to have an increased risk of hemorrhage. ${ }^{15,16}$ It is probable that small AVMs located at the cerebral convexity in relation with large subarachnoid spaces or in proximity to the ventricular system might be more susceptible to develop SS.

Superficial siderosis is a progressive disease for which no effective medical treatment is currently available. Administration of trienterine, a copper chelator, or desferrioxamine, an iron chelator, has shown little impact on the progression of SS. ${ }^{2,5}$ The evaluation of an underlying potentially treatable cause should be thorough in all cases. Surgical resection of the potential bleeding source may arrest recurrent SAH and normalize CSF after surgery. ${ }^{17,18}$ Being a chronic illness, the effectiveness of surgery in stabilizing or improving the symptomatology may not be seen before several years. Furthermore, it is unknown if a point exists at which the pathological process continues even after the bleeding source is eradicated. Interestingly, our patient has reported subjective improvements of his ataxia and urinary disturbances in the weeks following surgery.

In summary, cerebral AVMs are an infrequent cause of SS. AVMs of small size and located at the cerebral convexity in relation with large subarachnoid spaces or in proximity of the ventricular system might possibly be more susceptible to develop SS. Surgical resection of the AVM may stabilize this chronic illness and improve patient's symptomatology.

\section{REFERENCES}

1. Durieux A, Flocard F, Ferreira A, Azulay JP, Felten O, Navarro V, et al. Hémosidérose du système nerveux central. Rev Neurol. 1999;155(3):201-7.

2. Fearnley JM, Stevens JM, Rudge P. Superficial siderosis of the central nervous system. Brain. 1995;118(Pt 4):1051-66.

3. Offenbacher H, Fazekas F, Schmidt R, Kapeller P, Fazekas G. Superficial siderosis of the central nervous system: MRI findings and clinical significance. Neuroradiology. 1996;38 Suppl 1: S51-6.

4. Haroun RI, Li KW, Rigamonti D. Surgical resection of a cerebral arteriovenous malformation for treatment of superficial siderosis: case report. Surg Neurol. 2000;53(6):554-8.
5. Koeppen AH, Dentinger MP. Brain hemosiderin and superficial siderosis of the central nervous system. J Neuropathol and Exp Neurol. 1988;47(3):249-70.

6. Parnes SM, Weaver SA. Superficial siderosis of the central nervous system: a neglected cause of sensorineural hearing loss. Otolaryngol Head Neck Surg. 1992;107(1):69-77.

7. Bracchi M, Savoiardo M, Triulzi F, Daniele D, Grisoli M, Bradac GB, et al. Superficial siderosis of the CNS: MR diagnosis and clinical findings. AJNR. 1993;14(1):227-36.

8. McGee DA, Van Patter HJ, Morotta J, Olszewski J. Subpial cerebral siderosis. A report of two cases. Neurology. 1962;12(2):108-13.

9. Cammermeyer J. Deposition of iron in paraventricular areas of human brain in hemochromatosis. J Neuropathol Exp Neurol. 1947;6(2):111-27.

10. Hughes JT, Oppenheimer DR. Superficial siderosis of the central nervous system: a report of nine cases with autopsy. Acta Neuropathol. 1969;13(1):56-74.

11. Hashimoto M, Hoyt WF. Superficial siderosis and episodic fourth nerve paresis. Report of a case with clinical and magnetic resonance imaging findings. J Neuro-Ophtalmol. 1996; 16(4):277-80.

12. Hsu WC, Loevner LA, Forman MS, Thaler ER. Superficial siderosis of the CNS associated with multiple cavernous malformations. Am J Neuroradiol. 1999;20(7):1245-8.

13. Li KW, Haroun RI, Clatterbuck RE, Murphy K, Rigamonti D. Superficial siderosis associated with multiple cavernous malformations: report of three cases. Neurosurgery. 2001; 48(5): 1147-50.

14. Leussink VI, Flachenecker P, Brechtelsbauer D, Bendszus M, Sliwka U, Gold R, et al. Superficial siderosis of the central nervous system: pathogenetic heterogeneity and therapeutic approaches. Acta Neurol Scand. 2003;107(1):54-61.

15. Graf CJ, Perret GE, Torner JC. Bleeding from cerebral arteriovenous malformations as part of their natural history. $\mathrm{J}$ Neurosurg. 1983;58(3):331-7.

16. Kader A, Young WL, Pile-Spellman J, Mast H, Sciacca RR, Morh $\mathrm{JP}$, et al. The influence of hemodynamic and anatomic factors on hemorrhage from cerebral arterio-venous malformations. Neurosurgery. 1994;34(5):801-8.

17. Bonito V, Agostinis C, Ferraresi S, Defanti CA. Superficial siderosis of the central nervous system after brachial plexus injury. J Neurosurg. 1994;80(5):931-4.

18. Matsumoto S, Kang Y, Sato S, Kawakami Y, Oda Y, Araki M, et al. Spinal meningeal melanocytoma presenting with superficial siderosis of the central nervous system. Case report and review of the literature. J Neurosurg. 1998;88(5):890-4. 